\title{
Inclusion-to-specimen Volume Ratio Influence on the Strength and Stiffness Behaviors of Concrete: an Experimental Study
}

\author{
HAN Aylie ${ }^{1, a^{*}}$, BUNTARA Sthenly Gan ${ }^{2, b}$, RUDI Yuniarto ${ }^{3, c}$, \\ ANASTASIA Yesica ${ }^{4, d}$ and RAKHMA N. Editia ${ }^{4, e}$ \\ ${ }^{1}$ Associate Professor, Civil Engineering Department, Diponegoro University, Semarang, Indonesia \\ ${ }^{2}$ Professor, Department of Architecture, College of Engineering, Nihon University, Koriyama, Japan \\ ${ }^{3}$ Lecturer, Civil Engineering Department, Diponegoro University, Semarang, Indonesia \\ ${ }^{4}$ Research Assistant, Structural and Material Laboratory, Diponegoro University, Semarang, \\ Indonesia \\ ahanaylie@hccnet.nl, bbuntara@arch.ce.nihon-u.ac.jp, crudi_ya@yahoo.co.id, \\ danastasia.yesica@gmail.com, ${ }^{\mathrm{d}}$ rakhmanureditia@gmail.com
}

Keywords: inclusion-to-specimen volume ratio, compressive strength, stiffness, cracks propagation.

\begin{abstract}
The stress-strain response of the basic concrete making material, i.e. the mortar and aggregates, are well known. In general, the aggregate behaves linearly up till failure, possessing a very high ultimate compression strength and stiffness. The behavior of mortar is non-linear, even at low loading levels. The resulting composite material, the concrete, exhibits a less stiff response, in combination with degradation in strength. This study looked into the influence of the inclusion-tospecimen volume ratio of a 100x100x50 mm mortar specimen. Two inclusion configurations were considered, parallel and diagonal to the line of loading, while the ratio varied from zero to 0.66 . It was shown that the inclusion-to-specimen volume ratio strongly influenced the strength, the stiffness, and failure mode. The strength behavior had a minimum and a maximum bifurcation point, while the stiffness response increased, as a function of an increase in the inclusion-tospecimen volume ratio. Visual observation of the cracking pattern revealed that the initial cracking was always situated at the interface between the aggregate and mortar in tension and propagated through the mortar matrix. It was also perceived that the crack propagation path of the very dense, diagonally arranged inclusions deviated from the columnar configuration observed from the parallel inclusion formation. These densely diagonally arranged aggregates also resulted in spalling in the lateral direction.
\end{abstract}

\section{Introduction}

The behavior of concrete is a contribution of the mechanical properties of basic materials. Additionally, the characteristics of the interfacial transition zone (ITZ) between the aggregates and the mortar strongly influence the strength, stiffness and failure mode of the concrete. The aggregates have significantly higher compression strength in combination with an almost perfect linear stress-strain response up till failure. The stiffness modulus of most aggregates is also substantially larger when compared to the initial stiffness of the mortar matrix. The mortar matrix, on the other hand, exhibits a non-linear response, even at very low-stress levels. Its ultimate compression strength is approximately one-fifth of the aggregates while the initial stiffness modulus measured only one-seventh to the aggregates. These disparities in mechanical properties create stress concentrations and generate strain discrepancies in the boundaries between the inclusions and the mortar that will, in turn, promote premature micro crack initiation in the ITZ [1]. It was found that lessen these disparities lead to a delay in micro crack initiation in the ITZ.

The effect of aggregate shape on the stress concentrations was studied [2], and it was shown that the presence of sharp-edged inclusions significantly influenced the load carrying capacity. This negative effect is predominant when the sharp edge is situated in the line of the compression load. 
Studies on the influence of cylindrical inclusions to the concrete behavior were conducted involving single-inclusion specimens with a variation in diameter [3], and specimens with multiinclusions [4-6]. The research covered experimentally tested specimens, as well as finite element modeling. It was found that the ultimate load carrying capacity of a specimen was highly influenced by the inclusion-to-specimen volume ratio. The study conducted by Suarjana [4] and Han [3] suggested that the addition of a much stiffer inclusion resulted in a decrease in compression strength. Both studies concluded that the compression strength as a function of an increase in inclusion-tospecimen volume ratio followed a quadratic, convex path where at some point a minimum is reached. This pattern was similar in both studies, regardless of the fact that in the experiments conducted by Suarjana [4], the number of inclusions was gradually increased, and in the experiments carried out by Han [3] the diameter of cylindrical inclusion was enhanced.

This research looked into the effect of multiple cylindrical inclusions to the stiffness behavior, compressions strength response and crack pattern of a 100x 100x50 mm specimen. The decision on the dimensions were based on previous researches [7,8] while taking into consideration the plainstress analyzes for further finite element analysis purposes. Since it was shown that the configuration of aggregates also impacted the behavior of the specimen [6], the study was expanded to analyze the influences of inclusion formation on the strength, stiffness and crack pattern.

\section{Experimental Study}

The specimens were divided into two groups: the first was the inclusion configuration parallel to the line of loading designated $\mathrm{SL}_{\mathrm{i}}$, and the second was a diagonal inclusion arrangement labeled $\mathrm{SD}_{\mathrm{i}}$. The subscript $i$ was assigned to distinguish the inclusion-to-specimen volume ratio of the specimen. A mortar-only controlling specimen was also prepared and designated SO (Table 1).

For each group, six valid data were extracted. The specimens were cured through submerging and tested at the age of 28 days. The cylindrical inclusions were $20.8 \mathrm{~mm}$ in diameter and obtained by core-drilling natural stones. The aggregate cylinders were cut to a length of $50 \mathrm{~mm}$ to match the thickness of the specimen. The aggregate was volcanic in origin. The petro graphic study on the aggregate showed a small amount of chlorite $(2 \%)$, which is a secondary mineral originating from the deterioration process of the pyroxene minerals. The degree of deterioration strongly affects the bond between the aggregate and the mortar matrix, thus further having the potential of reducing the strength and stiffness of concrete [9].

Table 1 Specimen Details

\begin{tabular}{cccccccc}
\hline Specimen & $\mathrm{SO}$ & $\mathrm{SL}_{1}$ & $\mathrm{SL}_{2}$ & $\mathrm{SL}_{3}$ & $\mathrm{SD}_{1}$ & $\mathrm{SD}_{2}$ & $\mathrm{SD}_{3}$ \\
\hline Volume Ratio & 0 & 0.20 & 0.30 & 0.65 & 0.20 & 0.30 & 0.66 \\
\hline $\begin{array}{c}\text { Aggregate } \\
\text { Configuration }\end{array}$ & & $\mathbf{0} 0$ & $\mathbf{8 0 0}$ & 0000 & 0 & 0 & 0 \\
\hline
\end{tabular}

Prior to testing the specimens were dried and leveled to obtain a flat testing surface. The compression load was applied by a servo, load-controlled apparatus, and the load increment was set at $0.2 \mathrm{MPa} / \mathrm{sec}$. The load and displacement responses were recorded by a load cell and four LVDTs connected to a computer. Two Teflon sheets divided by a layer of grease were placed on top and at the bottom of the specimen to minimize the confining effect due to friction between the specimen's surface and the loading platen.

For the visual analysis of crack initiation and propagation, a high-definition camera was used to record the fracture process and observe the influence of the inclusion formation to the crack propagation. 


\section{Results and Discussion.}

The results of this study were categorized into quantitative and qualitative data. The quantitative data consist of the load-displacement response, the ultimate load carrying capacity and the specimen's initial stiffness. The qualitative data are the visual observation to the crack pattern and the examination of the differences in failure mode for each group of test specimens. The mortar matrix had a cylindrical compression strength $f^{\prime}{ }_{c}$ of $32.3 \mathrm{MPa}$ in combination with a Young's modulus of $23.7 \mathrm{GPa}$ while the aggregate had an ultimate compression strength of $148.8 \mathrm{MPa}$ with a constant stiffness modulus of $175.0 \mathrm{GPa}$. Fig. 1 shows the load-displacement responses of the SL and SD specimens with respect to the mortar-only specimen SO. The load-displacement responses of all specimens were recorded up till failure. The post-peak branch of the curve was not considered since at this stage the stiffness and strength, rather than the ductility, was of main interest. The loaddisplacement pattern followed a quadratic function for all specimens, with a very mild deviation in stiffness at every increment of the loading stages. This quadratic tendency of the load-displacement behavior became more pronounced as a function of increase in inclusion-to-specimen volume ratio. This can be clearly seen for specimens SD3 and SL3.
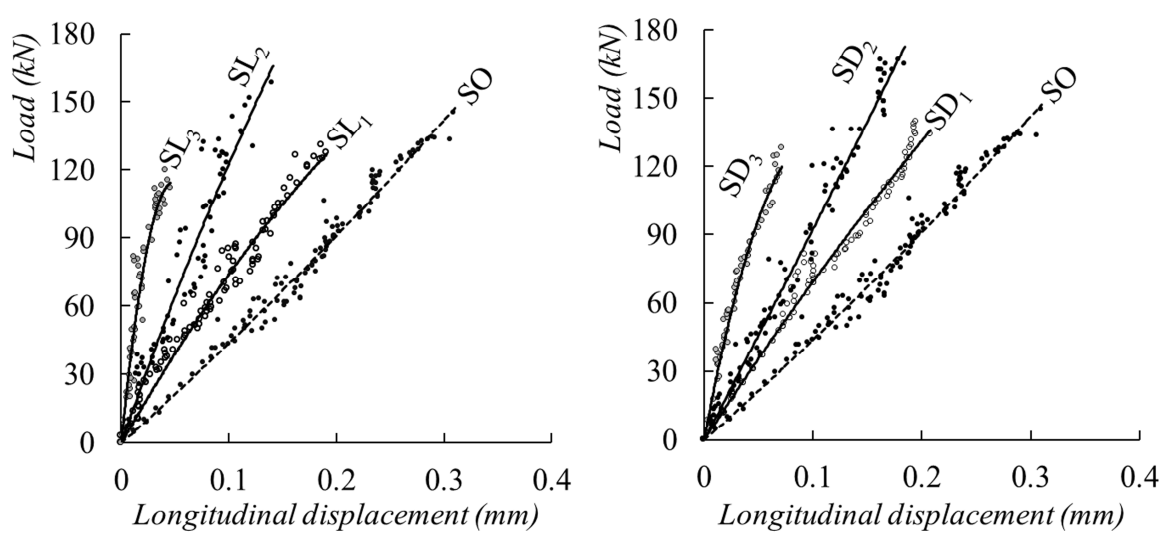

Fig. 1 Load-displacement response for specimens SL and SD with respect to SO

The Ultimate Load Response. The average of six valid data for each specimen category was calculated to represent the behavior of that particular group. The ultimate loads and initial stiffness of the specimens were further normalized to the data of the specimen without an inclusion, i.e., the mortar-only specimen SO, to ease the evaluation process. The normalized data of the SL as well as SD specimens were plotted against the inclusion-to-specimen volume ratio. The higher this ratio, the denser the aggregate inclusions with respect to the mortar. A ratio of zero was set for the mortar-only specimen SO to simulate the absence of inclusions. The resulting data is presented in Fig. 2.
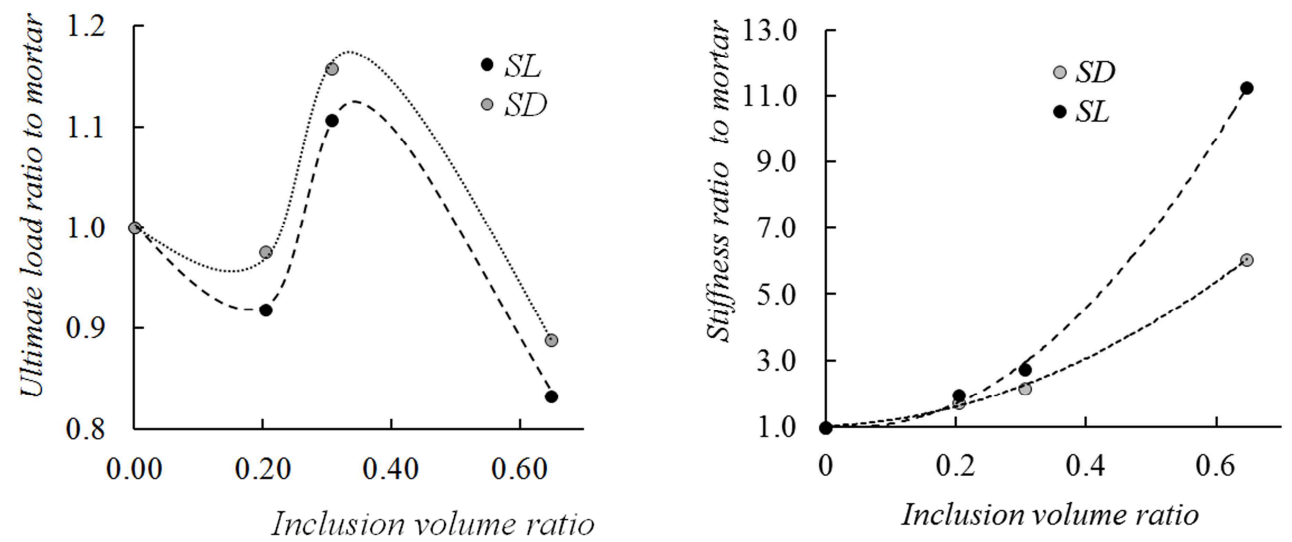

Fig. 2 The ultimate load and stiffness response to the inclusion-to-specimen volume ratio 
The graphs showed a consistent mode for both the parallel and diagonal inclusion configuration. The addition of aggregates with a volume ratio of 0.2 firstly resulted in a mild strength decrease. These findings underlined the findings of former researchers that the presence of inclusions will firstly result in a degradation of strength [4,10-11]. However, when the inclusion-to-specimen volume ratio was multiplied by $50 \%$, a compression strength increase was detected. At this point, the ultimate load for both the configurations SL and SD exceeded the load carrying capacity of the mortar-only specimen SO. Although the diagonal configuration SD resulted in a slight shifting of the curve to SL, the pattern of the both configurations was similar. The diagonal inclusion configuration SD exhibited a slightly higher load carrying capacity with a differentiation that ranged from $4.6 \%$ to $6.7 \%$ to the specimens SL. It is suggested that the behavior possesses two bifurcation points that correspond to minimum and maximum compression strength. For the specimens $\mathrm{SL}_{3}$ and $\mathrm{SD}_{3}$ with an inclusion-to-specimen volume ratio of 0.66 , the inclusions were placed very close together, and the compression strength dropped drastically.

Specimen Stiffness Behavior. The increase in inclusion-to-specimen volume ratio resulted in an enhancement in initial stiffness, confirming the findings in $[5,11]$ which shows that an increase in inclusion diameter results in an increase in specimen stiffness.

At low inclusion-to-specimen volume ratios, the stiffness of specimens SL and SD closely approached each other. However, as this ratio increased, it was seen that the parallel arranged inclusions $\mathrm{SL}_{2}$ and $\mathrm{SL}_{3}$ exhibited a much higher stiffness when compared to their counter parting diagonal specimens $\mathrm{SD}_{2}$ and $\mathrm{SD}_{3}$. The increase in stiffness was $26 \%$ and $86 \%$ respectively for $\mathrm{SL}_{2}$ and $\mathrm{SL}_{3}$. The increase response followed a parabolic, convex path.

Crack Pattern and Stress Concentrations. The visual behavior of specimens under increment loading was recorded and aimed to analyze the initial cracking and propagation toward fracture. Fig. 3 shows the initial cracking of specimen $\mathrm{SO}, \mathrm{SL}_{3}$ and $\mathrm{SD}_{3}$. For the mortar-only specimen $\mathrm{SO}$, a vertical crack was initiated almost simultaneously along the height of the specimen. This pattern agreed with the mechanics of the principal strains induced by the presence of the Poisson's ratio. Since confinement on top and at the bottom of the specimen was eliminated, the compression load resulted in lateral stresses and strain, which in turn induced longitudinal tensile strains. The corresponding tensile stresses in the direction perpendicular to the line of loading initiated the cracking in the mortar.

When inclusions were inserted into the mortar, the mode of failure shifted to the ITZ, being the weakest part of the concrete. It can be observed from Fig. 3 that for both $\mathrm{SL}_{3}$ and $\mathrm{SD}_{3}$ the first cracks appeared at the interface in tension. The cracks further propagated along the mortar, connecting the failed ITZ points. When the load was increased, the cracks multiplied until the specimen collapsed, as can be seen in Fig. 3.

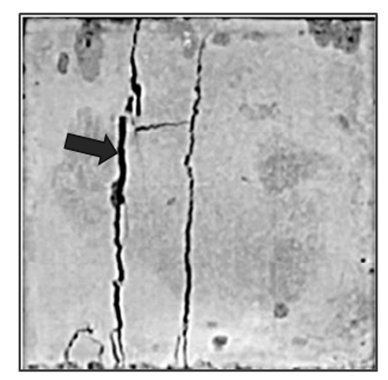

$\mathrm{SO}$

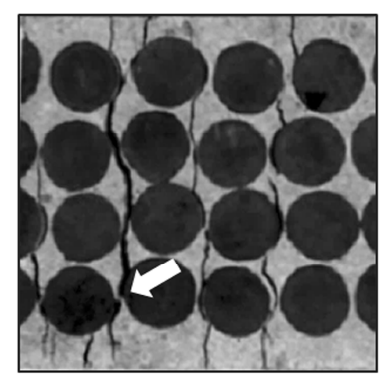

$\mathrm{SL}_{3}$

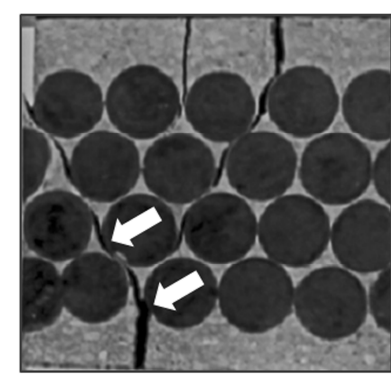

$\mathrm{SD}_{3}$

Fig. 3 Initial cracking and fracture mode of specimen

Prior to failure, it was seen that the cracks in the mortar-only specimen propagated vertically, parallel to the line of load. The number of fracture lines intensified, reaching the extreme top and bottom fibers, and the specimen failed due to tensile fracture. No mortar crushing was detected. For the inclusion specimens, the failed ITZ triggered the failure of the mortar, and multiple, vertical fracture lines were detected both for the $\mathrm{SL}_{3}$ and $\mathrm{SD}_{3}$ specimens. For the SL specimens, the arrangement of inclusions enabled the cracks to propagate along a relatively straight line, while for 
the $\mathrm{SD}_{3}$ specimens the crack propagation was diverted by the presence of the aggregate that were stacked pyramid-wise.

The deviation in the fracture pattern exhibited by $\mathrm{SD}_{3}$ was not seen in specimens $\mathrm{SD}_{1}$ and $\mathrm{SD}_{2}$ that both failed in a columnar mode (Fig. 4), emphasizing that the arrangement of inclusions with respect to the vertical axes determined the fracture pattern. All the $\mathrm{SD}_{3}$ specimens underwent spalling in the mortar, followed by spalling of the edge-inclusions prior to collapsing of the specimen. Surprisingly, the specimens $\mathrm{SD}_{1}$ and $\mathrm{SD}_{2}$ did not demonstrate any spalling, even when the ultimate load was reached. All parallel arranged inclusion specimens SL had no spalling, for all inclusion-to-specimen volume ratios.

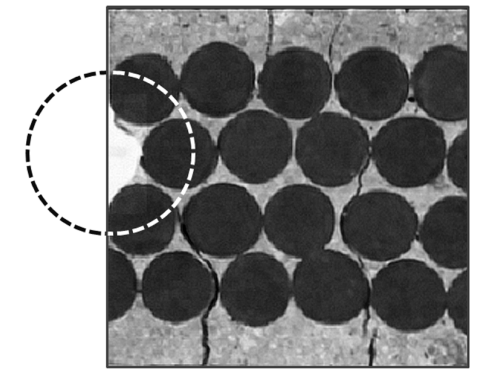

$\mathrm{SD}_{3}$

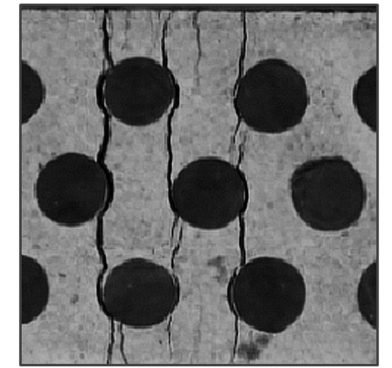

$\mathrm{SD}_{2}$

Fig. 4 Spalling and columnar failure mode for SD specimens

Analysis. The reduction in compression strength for the inclusions with a volume ratio of 0.2 is due to the presence of the ITZ. When inclusions are introduced, the specimen becomes a threephase, rather than a two-phase material, consisting of the mortar, the aggregates, and the ITZ. Although the aggregates have a higher strength and stiffness, the negative effect of the ITZ is predominant, and determined the final compression strength of the specimen. The stiffness, however, increased substantially, suggesting that the presence of the ITZ's not significantly influence the overall stiffness behavior of the specimen. This analysis is strengthened by the visual observation of the crack initiation. For both the specimens SL and SD, the first cracks always occur in the ITZ in tension, situated in a line perpendicular to the load line.

When the volume ratio is multiplied, the compression strength and stiffness increased. At this point, the negative effect of the weak ITZ is overshadowed by the presence of the strong inclusions. The initial crack location remains unchanged, and always occurs in the ITZ in tension. The failure crack pattern remained columnar, since the configuration of aggregates enabled the cracks to connect and propagate in a line parallel to the load. The diagonally placed inclusions had little effect on both the strength and stiffness of the specimen, compared to the parallel arranged group. It was found that for a 0.3 inclusion-to-specimen volume ratio an optimum in load carrying capacity was achieved. At this ratio, sufficient space in between the inclusions is present in the mortar to form a good bond with the inclusion.

For an inclusion-to-specimen volume ratio of 0.65 it was seen that the ultimate load decreased substantially. The explanation lay in the fact that the inclusions are so close together that the area of the already weak ITZ is reduced drastically, resulting in a very poor bond between the mortar and the aggregates. Furthermore, due to its very dense configuration, compacting becomes very difficult. $30 \%$ of the specimens showed honeycombs and large voids in the mortar. The initial stiffness of the specimen, recorded when the bond in the ITZ is preserved, was very high, approaching the stiffness of the aggregates. The SD specimens underwent spalling prior to failure, due to the tensile strains in the lateral direction. The crack pattern also deviated since a columnar crack path was diverted by the pyramid-wise arranged inclusions. The behavior underlined the study of Carpinteri [12] asserting the theory of stress concentrations induced by the presence of inclusions, which suggested that crack formations in material with a differentiation in strength are affected by the configuration of inclusions. 


\section{Conclusion}

The study of inclusion-to-specimen volume ratio concluded that the strength of concrete is a direct function of the aggregate-to-specimen volume ratio. Concrete is a three-phase element, and the synergy between the weak ITZ and strong aggregates resulted in a positive and negative optimum compression strength. It is therefore crucial in concrete mix design to determine the optimum percentage of coarse aggregates in the mix. The use of excessive aggregates with respect to the mortar has a negative influence on the compression strength and compounds the compaction process.

The initial stiffness of concrete is solely determined by the aggregate-to-specimen volume ratio. The load-displacement responses suggested that a high aggregate-to-specimen volume ratio results in a brittle material, which is not favored in construction. The use of fibers could enhance the ductility, and alter the failure mode of concrete. Research into this area is conducted at presence.

\section{References}

[1] T.W. Bremner and T.A. Holm, Elastic, compatibility and the behavior of concrete, ACI Mat. J. 83(2) (1986) 244-250.

[2] A.L. Han, J. Nurhuda and Y. Setiawan, The Effect of Aggregate Shape and Configuration to the Concrete Behavior, Smart Sci. 2(2) (2014) 85-90.

[3] A.L. Han, B.S. Gan and Y. Setiawan, The influence of single inclusions to the crack initiation, propagation and compression strength of mortar, Proc. Eng. 95 (2014) 376-385.

[4] M. Suarjana, M.S. Besari and R. Abipramono, Two-Dimensional Concrete Models Using Metal Aggregates, in: A. Katz and others (Eds.), RILEM Second International Conference on the Interfacial Transition Zone in Cementitious Composites, E \& FN Spon, London; New York, 1998, pp. 67-74.

[5] A.L. Han, S. Tudjono and J. Purnomo, Modeling the Load-Displacement Response of Concrete with Multi-Inclusion-Cylindrical Aggregates incorporating the Aggregate-Distance Effect and Configuration, $13^{\text {th }}$ East Asia-Pacific Conference on Structural Engineering and Construction (EASEC-13), September 11-13, 2013, Sapporo, Japan.

[6] A.L. Han, B.S. Gan and Y. Setiawan, The Aggregate Multi-inclusion Interaction and Interface Influence on the Compression Behavior of Concrete, The $8^{\text {th }}$ International Structural Engineering and Construction Conference (ISEC-8), November 23-28, 2015, Sydney, Australia. (accepted)

[7] I. Rasoolan, S.A. Sadrnejad and A.R. Bagheri, A Geometrical Inclusion-Matrix Model for Concrete, Proc. Struc. Cong., ASCE Lib. (2009) 1-10.

[8] K. Zhou, H.J. Hoh, X. Wang, L.M. Keer, J.H.L. Pang, B. Song and Q.J. Wang, A review of recent works on inclusions, Mech. Mat. 60 (2013) 144-158.

[9] J.J. Zheng, X.Z. Zhou and X.Y. Jin, An $n$-layered spherical inclusion model for predicting the elastic moduli of concrete with inhomogeneous ITZ, Cem. Conc. Comp. 34(5) (2012) 716-723.

[10] D. Darwin, Concrete in Compression, Conc. Int., ACI J. 21(8) (1999) 82-85.

[11] T. Akçaoğlu, M. Tokyay and T. Çelik, Effect of Coarse Aggregate Size and Matrix Quality on ITZ and Failure Behavior of Concrete under Uniaxial Compression, Cem. Conc. Comp. 26(6) (2004) 633-638.

[12] A. Carpinteri, Interpretation of the Griffith Instability as a Bifurcation of the Global Equilibrium, in: S.P. Shah (Ed.), Application of Fracture Mechanics to Cementitious Composites, Springer, Netherlands, 1985, pp. 287-316. 